\title{
Ergonomic Analysis of the Automotive Head-Up Displayed Information
}

\author{
Chung-Kee Yeh ${ }^{1, a}$, Yei-Po Fang ${ }^{1, b}, K^{2}$ uang-Yi Shih ${ }^{1, c}$ and Maxwell Jiang ${ }^{2, d}$ \\ ${ }^{1}$ Department of Bio-Industrial Mechatronics Engineering, National Taiwan University, Taiwan \\ ${ }^{2}$ Automotive Research and Testing Center, Taiwan \\ ackyeh@ntu.edu.tw, br00631001@ntu.edu.tw, 'r00631029@ntu.edu.tw, delem1017@artc.org.tw
}

Keywords: Head up display (HUD), Image, Visual information, Ergonomic analysis.

\begin{abstract}
A vehicle's head-up display (HUD) device enables the driver to obtain information, such as driving speed and engine conditions, to ensure safety while keeping the eyes focused on the front windshield. However, no complete and sufficient reference data are available regarding the extent of the driver's acceptability of the information displayed by such a device. Thus, this study aims to examine the driver's visual perception of the vehicular information pattern displayed by the HUD device and its influence on safe driving. The scope of this study includes the scope of the images displayed, quantity of information displayed, distribution and size of information, display brightness in different scenarios, suitable method of information display, correctness of interpretation, and so on. The researcher has built different scenarios for each item studied and analyzed the viewing effect through a questionnaire survey. A database can be built based on such data in the future. This database will contribute to the development of vehicle's display systems and verification of similar future studies.
\end{abstract}

\section{Introduction}

A head-up display (HUD) is an auxiliary device commonly used in aircrafts. The term "head-up" indicates that the operator can see necessary flight information without lowering his head. The HUD first appeared in military aircrafts to reduce the pilot's frequency of lowering his head to check the instrument as well as to avoid the interruption of the pilot's awareness and loss of control. In view of the HUD's convenience and safety features, civil aircrafts have been utilizing it. Several vehicle manufacturers now use a similar device, although the device has not been extensively applied yet.

General Motors began to install the HUD on its vehicles in 1988. Colored display appeared for the first time in the sports car Chevrolet Corvette C5 in 1998. Japanese-owned Toyota incorporated this device into its deluxe sedan Crown Majesta for domestic sale in 1991. BMW of Germany became the first manufacturer of vehicles with HUD in Europe in 2003. The HUD has been rapidly applied to vehicles; it generally displays the tachometer, speedometer, and navigation system of the vehicle. Several of the HUDs installed on GM, Honda, Toyota, and Lexus vehicles can display nighttime visual information. Other vehicle manufacturers, such as German Audi, French Citroen, Swedish Saab, and Japanese Nissan, also utilize certain types of HUD. [1-4]

\section{Purpose}

No complete and sufficient reference data are available regarding the extent of the driver's acceptability of the information displayed by the HUD. Thus, this study aims to examine the driver's visual perception of the vehicular information pattern displayed by the HUD and its influence on safe driving. This research also considers the scope of the images displayed, quantity of information displayed, distribution and size of information, display brightness in different scenarios, suitable method of information display, correctness of interpretation, and so on. The researcher has built different scenarios for each item studied and analyzed the viewing effect through a questionnaire survey. A database can be built based on such data in the future. This database will contribute to the development of vehicle's display systems and verification of similar future research. 


\section{Study Methods and Steps}

The ergonomic engineering analysis of visual information display mainly involves the evaluation of the visual comfort that the vehicle's display system provides to the human eyes and correctness of the information. The analysis is described below.

1. Evaluation of visual comfort: A scenario comprising six items, namely, the scope of the image displayed, quantity of information displayed, distribution of information, size of information, display brightness for different scenarios, and suitable method of information display is simulated for evaluation.

2. Information displayed versus correct interpretation by the driver: The scenario for evaluation is the driver's correct interpretation of the information on the display.

3. Ergonomic questionnaire survey: A questionnaire survey ( 300 questionnaire copies) is conducted to measure the actual experience provided by the abovementioned scenarios. A database can be built based on the relevant scenarios through statistical analysis of the questionnaire data.

The researcher installed a simulated testing platform in a laboratory. A windshield covered with heatproof film mounted on a fixed support was also installed. A flecting white board parallel to the ground was placed at the bottom of the windshield at a $45^{\circ}$ angle. Two projectors were placed above the white board. The projectors would cast a real image on this board, and people would see the virtual image on the windshield.

This study is carried out in the following steps:

1. Evaluation of visual comfort: The researcher evaluates the visual effect and comfort provided to human eyes through the ergonomic engineering method based on the driving habit and perspective of drivers. The researcher also determines the suitable scope of the images displayed (the $10 \mathrm{~cm} \times 20 \mathrm{~cm}$ full windshield), quantity of information displayed $(>2)$, distribution of information, size of information (clearly discernible at $60 \mathrm{~cm}$, corrected vision $>0.8$ ), display brightness (quality of display brightness at daytime, dusk, or nighttime) and method of information display (line, area, text, pattern, or other suitable types of information). A total of six items are evaluated.

2. Information displayed versus the driver's correct interpretation: The researcher evaluates the driver's interpretation of the displayed information. The simulation platform provides information, and the driver interprets the information and its meaning. The researcher determines the correctness of the interpretation of the information and evaluates the influence of each scenario on driving safety. 3. Questionnaire survey for ergonomic study: A total of 300 questionnaires are distributed. The selected respondents have driver's licenses and driving experiences. The average height of the respondents is $150 \mathrm{~cm}$ to $180 \mathrm{~cm}$. The gender ratio (male to female) is $2: 1$.

\section{Results and Discussion}

\section{Items on the Display for Evaluation}

Three, four, and five items of information are selected for display. These items are divided into two categories (with and without lane lines) for a total of six situations. The suitability of the graph of the information displayed and font size are considered. Font sizes that are too small are enlarged, and font sizes that are too large are reduced. The entire windscreen is considered the scope of the information displayed. The left half of the windscreen provides the image for left-hand drive vehicles; the right half of the windscreen provides the image for right-hand drive vehicles. The distribution of the information displayed is also considered (left top, left bottom, right top, or right bottom). When the information is distributed at the left bottom or left top and a lane line exists, the lane line must be at the central bottom of the windscreen and must overlap with the real lane line in front of the vehicle.

\section{Questionnaire Statistical Analysis}

The statistical analysis of the questionnaire addresses six items, namely, quantity of information displayed, size of information, scope of the image displayed, distribution of information, method of displaying information, and brightness of the scenario (daytime, dusk, and nighttime) based on the survey results of the male respondents, female respondents, and all of the respondents. 


\section{Quantity of Information Displayed}

The percentage of the quantity of information displayed for all respondents is shown through a pie chart in Fig. 1. Actually, all the three categories of respondents (male, female, and all respondents) prefer to display four pieces of information, including the lane line. The second preference is to display five pieces of information, including the lane line. The percentages of the four other scenarios are almost the same.

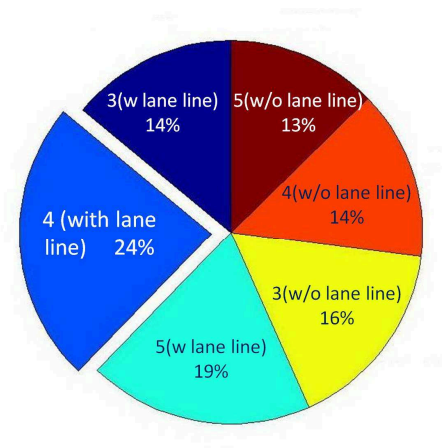

Fig. 1 Quantity of information displayed

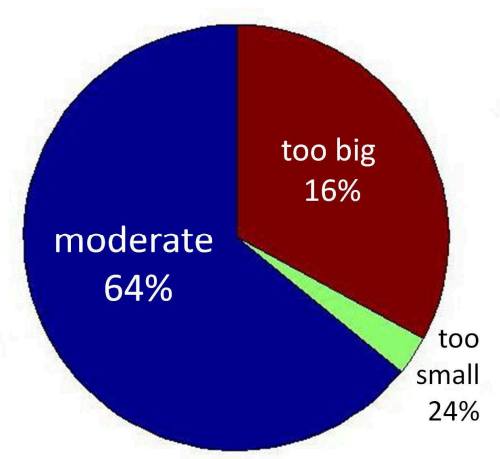

Fig. 2 Size of information

\section{Size of Information}

Fig. 2 shows the percentage in pie chart of the various sizes of information for all respondents. In fact, analysis of the pie chart reveals that more than $60 \%$ of the respondents in all three categories think that the size of the image is suitable, and only $30 \%$ of the respondents think that the display size is slightly larger and should be reduced.

\section{Scope of the Displayed Image}

Fig. 3 shows the percentage in pie chart of the scope of the displayed image selected by all respondents. Really, analysis of the pie chart reveals that more than $60 \%$ of the respondents in all three categories (male, female, and all respondents) think that the left half of the windshield can serve as the scope of the displayed image. The most probable reason for this finding is that domestic drivers sit on the left; thus, only $30 \%$ think that the entire windscreen should serve as the scope of the displayed image.

\section{Distribution of Information}

Fig. 4 shows the percentage in pie chart of the distribution of the information selected by all respondents. In reality, analysis of the pie chart reveals that more than $50 \%$ of the respondents in all three categories (male, female, and all respondents) think that the left bottom of the windshield is a suitable place to display the information. The left bottom of the windshield is also where most HUD's images are placed. Only $30 \%$ of the respondents think that the center bottom of the windshield is a suitable place to display information.

\section{Method of Displaying Information}

The percentage in bar graph of the various methods of displaying information selected by all respondents is shown in Fig. 5. Essentially, graphical analysis reveals that all three categories (male, female, and all respondents) prefer the original design of signals or digital signs, and only a few respondents prefer to use red for fuel level warning. Several respondents think that the brake warning is more important than the fuel level warning.

\section{Brightness of Scenario}

Fig. 6 shows the bar graphs of the brightness of the scenario (daytime, dusk, and nighttime) selected by all respondents. Virtually, graphical analysis reveals that most of the respondents in the three categories (male, female, and all respondents) think that the displayed items of information are 
clearly discernible in the simulated scenarios of daytime and dusk. As to the simulated nighttime scenario, more than half of the respondents think that the displayed items of information are extremely bright.

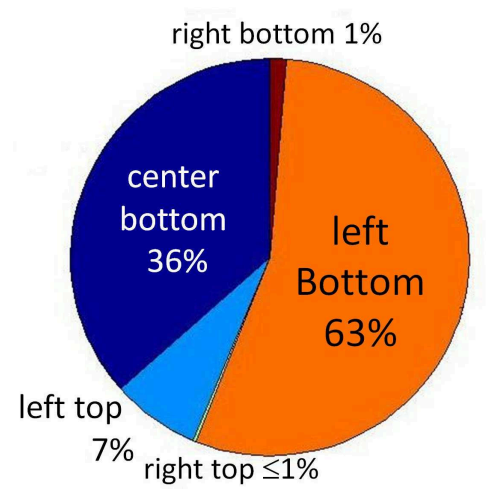

Fig. 4 Distribution of information
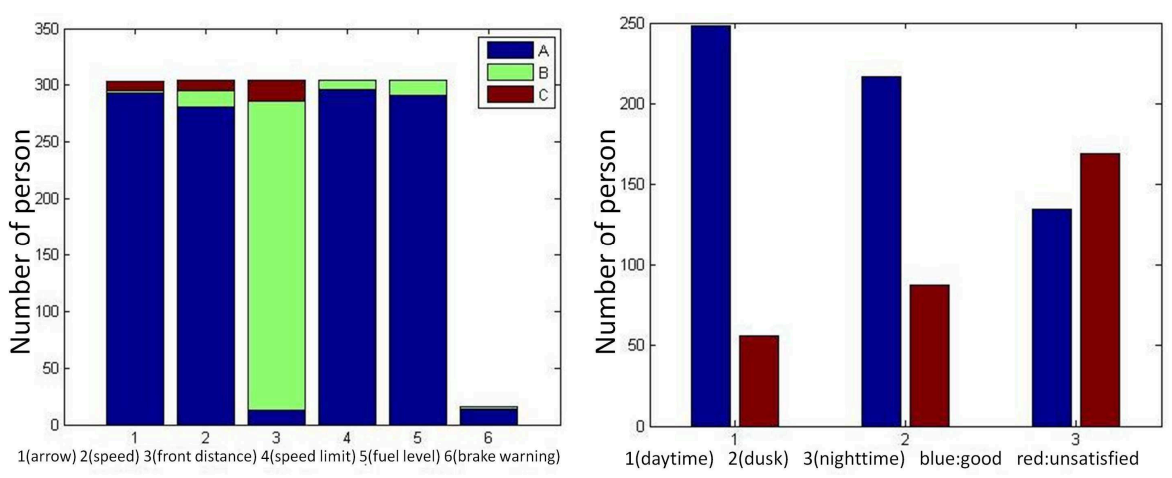

Fig. 5 Method of displaying information

Fig. 6 Display scenario

\section{Conclusions}

This study evaluates mainly the visual comfort provided by the HUD to human eyes based on the following factors: a) building a scenario for evaluation that consists of six items, including the scope of the image displayed, quantity of information displayed, distribution of information, size of information, display brightness for the different scenarios, and suitable method of displaying information, b) the information displayed versus the correct interpretation by the driver: building a scenario for evaluation that considers the driver's correct interpretation of the displayed information, and c) the ergonomic questionnaire survey: providing an actual simulation of the three aforementioned scenarios and conducting a questionnaire survey (a total of 300 copies). A database can be established based on the relevant scenarios through the statistical analysis of the questionnaire data. The analysis of the survey and database reveals that most of the respondents think that the scenario that provides better visual comfort is the one that has four items of information and includes the lane line, scope of the image displayed on the left half of the windshield, distribution of information on the left bottom side, suitable method of information display that includes the guidance arrow, driving speed, distance to the preceding vehicle, oil level, maximum speed limit, and other signals. The brightness of both daytime and dusk scenarios is acceptable. Only half of the respondents think that the nighttime brightness is extreme, and most of the respondents think that the lane line should be displayed.

\section{References}

[1] M. AblaJimeier, T. Poitschke, F. Wallhoff, K. Bengler and G. Rigoll, Eye gaze studies comparing head-up and head-down displays in vehicles, Multimedia and Expo, 2007 IEEE International Conference, 2007, pp. 2250-2252.

[2] K.W. Gish and L. Staplin, Human factors aspects of using head up display in automobiles: a review of the literature, U.S. Department of Transportation, 1995.

[3] Y.C. Liu and M.H. Wen, Comparison of head-up display (HUD) vs. head-down display (HDD): driving performance of commercial vehicle operators in Taiwan, International Journal of Human-Computer Studies 61 (2004) pp. 679-697.

[4] R.B. Wood and P.J. Howells, Head-up displays: a review of the literature, CRC Press LLC, 2001. 